\title{
CENTRAL EXCLUSIVE PRODUCTION MEASUREMENTS IN THE LHCb*
}

\author{
Mateusz Goncerz, Barteomiej Rachwae, Tomasz Szumlak \\ on behalf of the LHCb Collaboration
}

AGH University of Science and Technology, Kraków, Poland

(Received April 24, 2018)

\begin{abstract}
Central Exclusive Production (CEP) is a class of diffractive processes studied at the Large Hadron Collider, that offers a very clean experimental environment for probing the low-energy regime of Quantum Chromodynamics and testing the Standard Model predictions. The paper summarizes the $\mathrm{CEP}$ analyses conducted by the $\mathrm{LHCb}$ Collaboration. The latest measurements of $J / \psi$ and $\psi(2 S)$ photoproduction are discussed, along with the impact of the HeRSCheL detector on dissociation background reduction. Finally, a novel Monte Carlo event generator for diffractive physics is presented as a work-in-progress project.
\end{abstract}

DOI:10.5506/APhysPolB.49.1135

\section{Introduction}

Central Exclusive Production (CEP) is a class of $t$-channel specific diffractive processes with both protons surviving the collision. The interaction results in a very central system that, coupled with the outgoing protons, gives rise to large regions devoid of any activity, called rapidity gaps. This finalstate signal signature is exploited in the search of CEP event candidates.

Experimentally, it provides a very clean environment for the study of soft gluon interactions as well as setting the stage for glueball [1] searches.

\section{2. $\mathrm{LHCb}$ detector}

The LHCb detector [2] is a single-arm forward spectrometer that is fully instrumented in the pseudorapidity range $2<\eta<5$ with some additional backward tracking provided by the VELO subsystem. With the ability to

* Presented at the Cracow Epiphany Conference on Advances in Heavy Flavour Physics, Kraków, Poland, January 9-12, 2018. 
trigger on particles with very low transverse momenta (above $400 \mathrm{MeV}$ ) and very few interactions per bunch crossing (with about $20 \%$ of total collected luminosity being single interaction events), it is ideal for studying diffraction processes.

\section{LHCb Run 2 upgrade}

The main source of background for CEP processes is proton dissociation that leads to very forward activity due to particle showers close to the beam pipe. To exploit this, a set of five High Rapidity Shower Counters (HeRSCheL) with four scintillating pads each have been installed along the beam for Run 2 of the LHC [3].

By increasing the effective rapidity gap detection range to include $-10<$ $\eta<-5$ and $5<\eta<10$, the ability to veto inelastic interactions is drastically improved, leading to an approximate halving of the proton dissociation background compared to the $7 \mathrm{TeV}$ analyses [4].

\section{Completed and ongoing analyses}

Several analyses regarding Central Exclusive Production have been performed by the LHCb Collaboration (Table I). Most notably, the recent $J / \Psi$, $\Psi(2 S)$ photoproduction measurement with $13 \mathrm{TeV}$ data, introducing the novel HeRSCheL activity threshold requirement. Its details are discussed below [4]. There are also ongoing analyses that exploit both ion-proton (32.31 $\mathrm{nb}^{-1}$ of total integrated luminosity) and proton-proton $\left(3.71 \mathrm{fb}^{-1}\right)$ data. The former being particularly interesting for photoproduction study, because ions have higher probability of being the emitters of virtual photon which, in turn, removes the vertex ambiguity. Since the LHCb detector is a forward spectrometer, both ion-proton and proton-ion collisions are being conducted.

TABLE I

Completed LHCb analyses on Central Exclusive Production.

\begin{tabular}{l|ll}
\hline \hline \multicolumn{3}{c}{ Run 1} \\
\hline$J / \Psi, \Psi(2 S)$ photoproduction & $7 \mathrm{TeV}$ & {$[5]$} \\
Upsilon photoproduction & 7 and $8 \mathrm{TeV}$ & {$[6]$} \\
Double charmonium & $7 \mathrm{and} 8 \mathrm{TeV}$ & {$[7]$} \\
$\chi_{c}$ & $7 \mathrm{TeV}$ & {$[8]$} \\
Dimuon (QED) & $7 \mathrm{TeV}$ & \\
\hline \multicolumn{3}{c}{ Run 2} \\
\hline$J / \Psi, \Psi(2 S)$ photoproduction & $13 \mathrm{TeV}$ & {$[4]$}
\end{tabular}




\section{5. $J / \psi$ and $\psi(2 S)$ photoproduction at $13 \mathrm{TeV}$}

The analysis has been performed on $300 \mathrm{pb}^{-1}$ of data selected as single interaction events (achieving about 1.1 average visible interactions).

Two muons within the fully instrumented detector region $(2<\eta<4.5)$ were required with no other activity detected and the reconstructed dimuon mass to be within $65 \mathrm{MeV}$ of the known meson mass.

Additionally, a threshold has been set on the combined charged particle activity in the HeRSCheL scintillating pads, effectively halving the dissociation background as compared to the Run 1 measurements.

The total activity in HeRSCheL is expressed with a metric, defined as

$$
\Sigma_{H}=\sum_{i=1}^{20}\left(\frac{\mathrm{ADC}_{i}}{2.5 \mathrm{RMS}_{i}}\right)^{2},
$$

where the sum goes over each of the HeRSCheL pads. The $\mathrm{ADC}_{i}$ is the number of raw ADC counts and $\mathrm{RMS}_{i}$ is the root mean square in the empty detector signal, recorded after a bunch train [3].

Low values of the $\Sigma_{H}$ variable can be linked to the lack of dissociation in a given event. A clear distinction is visible between the CEP candidate events (low charged particle activity) and the background (Fig. 1).

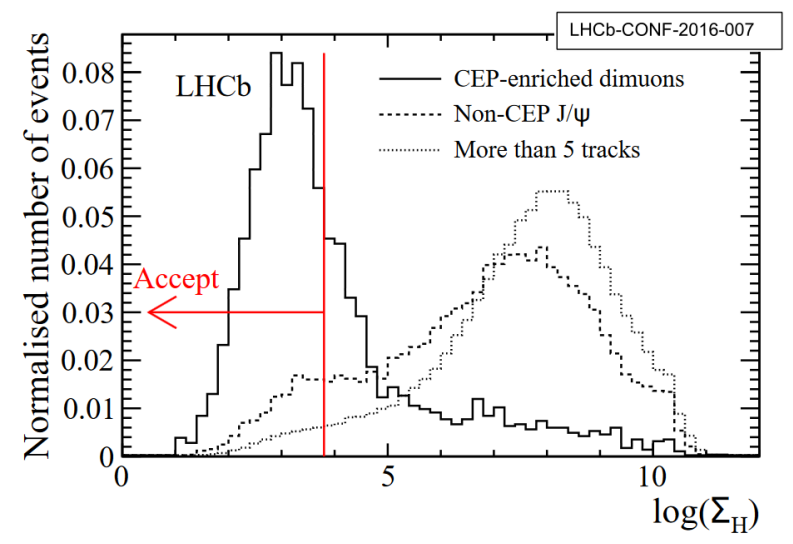

Fig. 1. Selection requirement on combined HeRSCheL activity [4].

The remaining background is estimated by fitting the transverse momentum squared distributions (Fig. 2). As mentioned before, the main source of the background is proton dissociation, in addition, the $\Psi(2 S)$ measurement shows a significant contribution from QED processes. The feed-down provides a small amount of background as compared with the dominating dissociation source and in the case of $\Psi(2 S)$, it becomes negligible (Table II). 

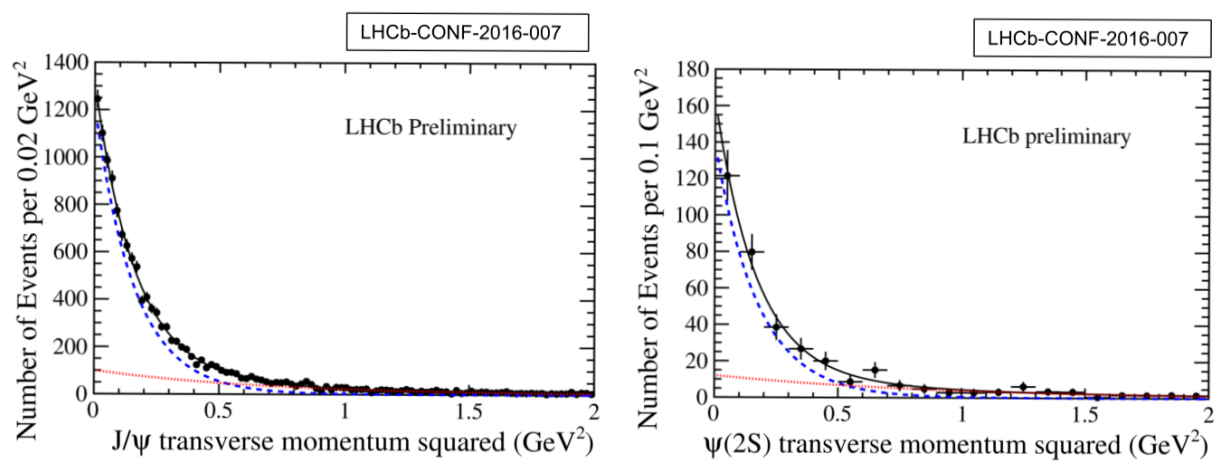

Fig. 2. Transverse momentum squared distribution of background-substracted $J / \psi$ (left) and $\Psi(2 S)$ (right) candidates [4].

TABLE II

Composition of background for $J / \psi$ and $\Psi(2 S)$ candidates.

\begin{tabular}{l|cc}
\hline \hline & $J / \psi$ & $\Psi(2 S)$ \\
\hline QED & $0.9 \%$ & $17.5 \%$ \\
Feed-down & from $\Psi(2 \mathrm{~S}), \chi_{c} 5.9 \%$ & negligible \\
Dissociation & $20 \%$ & $21 \%$
\end{tabular}
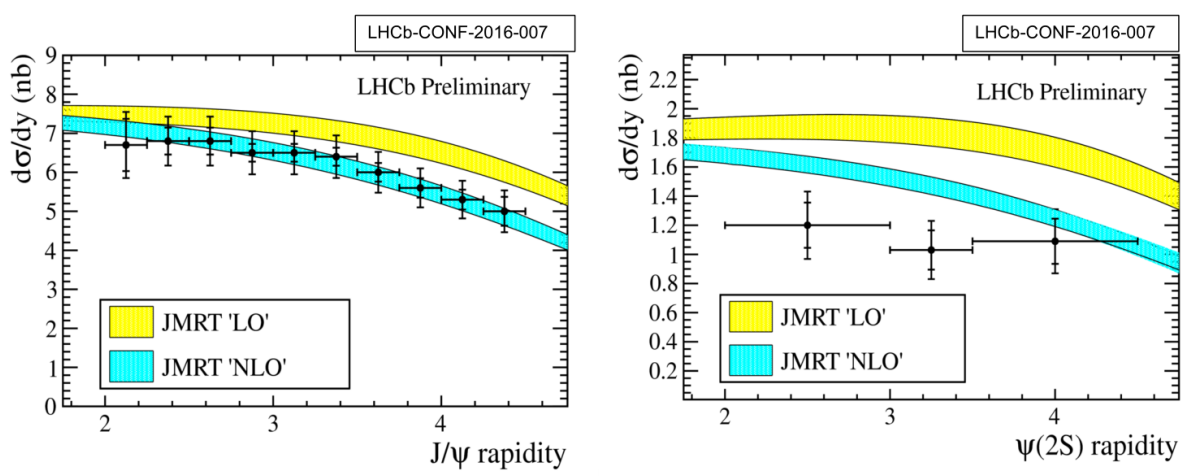

Fig. 3. Differential cross section compared to LO and NLO predictions. The inner error bar represents the statistical uncertainty; the outer is the total uncertainty. 
The measured cross sections for both muons within the required pseudorapidity region are

$$
\begin{aligned}
& -\sigma_{J / \Psi \rightarrow \mu^{+} \mu^{-}}\left(2.0<\eta_{\mu^{+}}, \eta_{\mu^{-}}<4.5\right)=(407 \pm 8 \pm 24 \pm 16) \mathrm{pb} \\
& -\sigma_{\Psi(2 S) \rightarrow \mu^{+} \mu^{-}}\left(2.0<\eta_{\mu^{+}}, \eta_{\mu^{-}}<4.5\right)=(9.4 \pm 0.9 \pm 0.6 \pm 0.4) \mathrm{pb}
\end{aligned}
$$

with uncertainties respectively: statistical, systematic and from the luminosity determination. In both cases, the Next Leading Order (NLO) calculation is preferred (Fig. 3).

\section{GenEx}

Since Central Exclusive Production involves mainly the low-energy regime of QCD and is thus based on rapidly changing phenomenological models, the use of general-purpose Monte Carlo event generators (e.g. Pythia8 [9]) tends to be cumbersome.

A GenEx [10] event generator for diffractive physics is being introduced to the $\mathrm{LHCb}$ software framework. Written in $\mathrm{C}++$, it is aimed at replacing the older generation of Fortran-based generators with a lightweight and modern package. The key idea is to provide the community with a user-friendly and effective way of testing new models and generating Monte Carlo samples.

Although being still in the work-in-progress stage, it is expected to be available soon along with a standalone version for use outside of the $\mathrm{LHCb}$ experiment. The processes that are expected to be included in the release version are

— resonant $\phi[11]$ and $J / \Psi[12]$ meson production with $K_{\mathrm{T}}$-factorization approach;

- $f_{0}(980) \rightarrow \pi \pi$ and non-resonant $\pi \pi$ production with tensor amplitudes [13].

\section{Summary}

The Central Exclusive Production analyses conducted by the LHCb Collaboration have been summarized and the results of $J / \psi$ and $\psi(2 S)$ photoproduction differential cross-section measurement with Run 2 dataset presented. The recent analysis includes the HeRSCheL activity threshold, reducing the background from proton dissociation.

Additionally, a new Monte Carlo event generator, based on the GenEx framework, is being introduced into the experiment along with a standalone version. 
This work was partially supported by the Faculty of Physics and Applied Computer Science AGH University of Science and Technology statutory tasks No. 11.11.220.01/4 and supported in part by the National Science Centre, Poland (NCN) grant UMO-2015/17/D/ST2/03530.

\section{REFERENCES}

[1] Y.M. Cho et al., Phys. Rev. D 91, 114020 (2015).

[2] A.A. Alves, Jr. et al. [LHCb Collaboration], JINST 3, S08005 (2008).

[3] K. Carvalho Akiba et al., JINST 13, P04017 (2018) [arXiv: 1801.04281 [physics.ins-det]].

[4] LHCb Collaboration, LHCb-CONF-2016-007, CERN-LHCb-CONF-2016-007.

[5] R. Aaij et al. [LHCb Collaboration], J. Phys. G 41, 055002 (2014) [arXiv: 1401.3288 [hep-ex]].

[6] R. Aaij et al. [LHCb Collaboration], J. High Energy Phys. 1509, 084 (2015) [arXiv: 1505.08139 [hep-ex]].

[7] R. Aaij et al. [LHCb Collaboration], J. Phys. G 40, 045001 (2013) [arXiv: 1301.7084 [hep-ex]].

[8] LHCb Collaboration, LHCb-CONF-2011-022, CERN-LHCb-CONF-2011-022.

[9] T. Sjöstrand et al., Comput. Phys. Commun. 191, 159 (2015) [arXiv:1410.3012 [hep-ph]].

[10] R.A. Kycia et al., arXiv:1711.06087 [hep-ph].

[11] A. Cisek, W. Schäfer, A. Szczurek, Phys. Lett. B 690, 168 (2010).

[12] A. Cisek, W. Schäfer, A. Szczurek, J. High Energy Phys. 1504, 159 (2015).

[13] P. Lebiedowicz, O. Nachtmann, A. Szczurek, Phys. Rev. D 93, 054015 (2016) [arXiv: 1601.04537 [hep-ph]]. 